\title{
Consistencia entre minimización de varianza y maximización de utilidad en la evaluación de derivados
}

Francisco Venegas

\section{Martínez}

Profesor investigador, Escuela Superior de Economía, Instituto Politécnico Nacional fvenegas1111@yahoo.com.mx

\section{Abigail Rodríguez}

Nava

Profesora investigadora, Departamento de Producción Económica, Universidad Autónoma Metropolitana-Xochilmilco arnava@correo.xoc.uam.mx

\section{Resumen}

Este artículo muestra la consistencia entre el postulado de racionalidad de Markowitz-Sharpe (MS) para resolver problemas de decisión sobre un portafolio de activos y el postulado de racionalidad económica de Pareto-Walras-Marshall (PWM) para resolver problemas de decisión sobre el consumo de una canasta de bienes, dada una restricción presupuestal que incorpora activos. Ambos postulados están basados en el comportamiento optimizador de los agentes. En el primer caso, los inversionistas minimizan la varianza del rendimiento de los activos de un portafolio sujeto a un rendimiento esperado. En el segundo caso, los consumidores maximizan utilidad por el consumo de un bien sujeto a una restricción presupuestal que incorpora los activos disponibles en la economía. La consistencia entre ambos postulados se muestra de la siguiente manera: en un ambiente de riesgo generado por activos, cuyos precios son conducidos por procesos de difusión, los dos postulados son utilizados para valuar una opción europea. El resultado principal es que en ambos casos se obtiene la misma ecuación diferencial parcial que caracteriza el precio de la opción, la cual coincide con la ecuación diferencial parcial de Black-Scholes-Merton (1973).

Palabras clave: Productos derivados, portafolios, comportamiento racional.

Clasificación JEL: G11, G13, D1. 


\title{
Consistency between minimization of variance and maximization of utility in pricing derivatives
}

\begin{abstract}
This paper shows the consistency between the Markowitz-Sharpe's rationality premise (MS) used to solve decision problems about the assets integrating a portfolio and the Pareto-Walras-Marshall's economic rationality premise (PWM) used to solve decision problems about the consumption of goods integrating a basket given a budget constraint incorporating assets. Both premises are based on the optimizing behavior of the agents. In the first case, investors minimize the variance of the assets returns of a portfolio subject to an expected return. In the second case, consumers maximize utility from the consumption of a generic good subject to a budget constraint incorporating the available assets in the economy. The consistency between the two postulates described above is shown as follows: under an environment of market risk generated by assets whose prices are driven by diffusion processes both postulates are used to value a European option. The main result is that both cases result in the same Black-ScholesMerton (1973) partial differential equation that characterizes the option price, which agrees with the partial differential equation.
\end{abstract}

Keywords: Derivates, portfolios, rational behavior.

JEL Classification: G11, G13, D1.

\section{Introducción}

Esta investigación examina la consistencia entre los postulados que utilizan los financieros y los economistas para abordar los problemas que a cada uno les interesan. Para los financieros las decisiones de consumo son, en general, irrelevantes, mientras que para los economistas son fundamentales. Por otro lado, la conformación de un portafolio de activos con ciertas características no es, en general, un asunto que seduzca a los economistas, pero sí a los financieros. Tanto en finanzas como en economía se establece en cada una de estas disciplinas un postulado esencial para su desarrollo teórico: en el primer caso, se propone el postulado de racionalidad de Markowitz-Sharpe para resolver problemas de decisión sobre los activos que deben integrar un portafolio y, en el otro caso, se plantea el postulado de racionalidad económica de Pareto-Walras-Marshall para resolver problemas de decisión sobre los bienes y cantidades por consumirse, sujetos a una restricción presupuestal que incorpora los activos disponibles en la economía. Estos postula- 
dos están basados en el comportamiento optimizador, también llamado comportamiento racional, de los agentes.

El postulado de racionalidad de Markowitz-Sharpe establece que un inversionista toma decisiones de portafolio de tal forma que minimiza la varianza del rendimiento de los activos de dicho portafolio sujeto a un rendimiento esperado. El portafolio puede incluir un bono libre de riesgo (de incumplimiento), activos riesgosos (acciones) e incluso productos derivados (futuros, opciones europeas, notas estructuradas, etc.) sobre dichos activos. En este caso, la varianza del rendimiento del portafolio es vista como una medida de "riesgo" de mercado. Por otro lado, el postulado de racionalidad económica de Pareto-Walras-Marshall establece que un consumidor toma decisiones de tal manera que maximiza su utilidad (satisfacción o felicidad) por el consumo de una canasta de bienes sujeto a su restricción presupuestal; esta restricción puede considerar la posibilidad de que el individuo adquiera activos disponibles en la economía.

El experimento que en este trabajo se llevará a cabo para mostrar la consistencia entre los dos postulados anteriores se describe a continuación. En un ambiente de riesgo generado por activos cuyos precios son conducidos por movimientos geométricos Brownianos (activos con rendimientos normales con media y varianza dependientes, linealmente, del tiempo) se analiza a un inversionista minimizador de varianza de los rendimientos de un portafolio sujeto a un rendimiento dado. Este modelo se empleará para valuar un derivado (una opción europea). Posteriormente, se considera un consumidor que maximiza utilidad sobre un bien genérico de consumo sujeto a una restricción presupuestal que incorpora activos. De igual manera, este modelo se usará para valuar el mismo derivado. El resultado principal es que en ambos casos se obtiene la misma ecuación diferencial parcial de segundo orden que determina la prima de una opción, a saber, la ecuación diferencial parcial de Black-Scholes-Merton (BSM), lo que muestra que los dos postulados mantienen cierta consistencia entre sí. Es importante destacar que esta investigación toma como punto de partida los trabajos de Markowitz (1952) sobre la construcción de portafolios; Sharpe (1964) sobre valuación de acciones; Black y Scholes (1973) y Merton (1973) acerca de la valuación de opciones; y Venegas-Martínez (2001), (2005), (2006) y (2008) sobre decisiones de consumo y portafolio (incluyendo derivados) en ambientes de riesgo e incertidumbre. 
En 1973, Fischer Black y Myron Scholes y, de manera independiente, Robert Merton desarrollaron, bajo el supuesto de ausencia de oportunidades de arbitraje (ley de un solo precio), un modelo para valuar una opción europea cuando el precio del activo subyacente es conducido por un movimiento geométrico Browniano (i.e., los rendimientos son normales con media y varianza escaladas por el tiempo). Una situación anecdótica relacionada con el trabajo de investigación de Fischer Black y Myron Scholes es que fue rechazado, primero, en 1970 por The Journal of Political Economy (JPE) de la Universidad de Chicago y, posteriormente, en 1971, por The Review of Economics and Statistics de la Universidad de Harvard. No obstante, después de incorporar varias recomendaciones sugeridas por Merton Miller y Eugene Fama, incluyendo el cambio del título tres veces de "A theoretical Valuation Formula for Options, Warrants and other Securities" a "Capital Market Equilibrium and the Pricing of Corporate Liabilities" y, finalmente, a "The Pricing of Options and Corporate Liabilities", el trabajo fue reconsiderado y, finalmente, aceptado por el "JPE" para ser publicado en 1973. El trabajo de Robert Merton "Theory of Rational Option Pricing" fue aceptado casi inmediatamente y publicado, también en 1973, en el Bell Journal of Economics and Management Science. Felizmente, 24 años después, en 1997, Robert Merton y Myron Scholes son laureados por la Academia Sueca de Ciencias con el premio nobel de economía; lamentablemente, el destacado matemático y economista Fischer Black había fallecido dos años antes.

El modelo BSM ha fomentado de manera importante que los participantes en los mercados financieros se cubran, conveniente y oportunamente, contra los diversos riesgos de mercado a que están expuestos, pero también ha promovido a que inviertan en fondos de derivados con fines especulativos. Evidentemente, el modelo BSM ha desempeñado un papel central en el impresionante avance que ha tenido la economía financiera y las matemáticas financieras modernas. Ante todo esto, es importante destacar que dicho modelo puede ser empleado como herramienta para generar ganancias de millones de dólares en periodos cortos (unas semanas), pero también si no se utiliza adecuadamente puede generar pérdidas astronómicas en periodos aún más cortos (unos días). La importancia práctica del modelo BSM se refleja en que su aparición es casi simultánea con el arranque de la bolsa de opciones "Chicago Board of Options Exchange" (Свот), la cual ha operado, a la fecha, cientos de billones de dólares (el Chicago Mercantile Exchange (CME) compró en julio de 2007 en 12.1 billones de dólares al свот en una estrategia de conformar el mercado de derivados, financieros y físicos, más grande del mundo). Asimismo, la aparición del modelo BSM aunado al asombroso avance de las tecnologías de 
información y a la sorprendente aparición de decenas de nuevas revistas de investigación en matemáticas financieras y administración de riesgos, obliga a reconocer la gran importancia de la contribución de Black, Scholes y Merton a la teoría, pero sobre todo a la práctica financiera.

El presente trabajo está organizado como sigue. En la sección dos se utiliza el modelo de Markowitz para obtener la ecuación diferencial parcial de BSM. En el transcurso de la sección tres se obtiene la ecuación de BSM bajo el supuesto de que existen consumidores que maximizan utilidad sobre un bien genérico sujetos a su restricción presupuestal. Por último, en la sección cuatro se presentan las conclusiones, así como las limitaciones y sugerencias para futuras investigaciones. Un apéndice contiene el lema de Itô útil en la obtención de diferenciales estocásticas.

\section{Obtención de la ecuación BSM mediante el modelo de Markowitz}

En esta sección se obtendrá la ecuación diferencial parcial de BSM mediante el modelo de Markowitz. Antes de bosquejar cualquier modelo o escribir cualquier ecuación es importante establecer los supuestos que delimitarán el marco y los alcances de este trabajo de investigación:

- los agentes tienen comportamiento optimizador;

- el activo subyacente es una acción que no paga dividendos durante la vida del contrato;

- el precio del activo subyacente es conducido por el movimiento geométrico Browniano, es decir, los rendimientos son normales con media y varianza escaladas por el tiempo;

- la volatilidad del precio del activo subyacente se mantiene constante a través del tiempo;

- el mercado del subyacente es líquido y divisible, es decir, el subyacente siempre se puede comprar y vender en cualquier fracción de unidad;

- no hay costos de transacción (comisiones e impuestos); 
- el mercado opera en forma continua, es decir, no hay fines de semana ni días festivos (en cada instante hay un precio);

- existe un mercado de crédito o un sistema bancario en el que los agentes pueden prestar y pedir prestado a una tasa de interés constante a todos los plazos y libre de riesgo de incumplimiento.

Una vez que se han establecido los supuestos básicos, se introducen ahora los aspectos técnicos necesarios para la construcción del modelo de Markowitz en un ambiente de riesgo modelado con procesos de difusión.

\section{Dinámica del precio del subyacente y riesgo de mercado}

Considere un movimiento Browniano $\left(W_{t}\right)_{t \in[0, T]}$ definido sobre un espacio fijo de probabilidad con su filtración aumentada $\left(\Omega, \mathcal{F},\left(\mathcal{F}_{t}^{W}\right)_{t \in[0, T]}, P\right)$. En este caso, el espacio medible en cuestión es $(\Omega, \mathcal{F}):=(\mathbf{R}, B(\mathbf{R}))$ donde $B(\mathbf{R})$ es la $\sigma$-álgebra estándar de Borel sobre $\mathbf{R}$. La medida de probabilidad satisface

$$
\mathrm{dP}(\omega)=\frac{1}{\sqrt{2 \pi t}} \exp \left\{\frac{\omega^{2}}{2 t}\right\} \mathrm{d} \omega .
$$

La filtración $\left(F_{t}^{W}\right)_{t \in[0, T]}$ es una familia de $\sigma$-álgebras tales que $F_{t} \subset F$ para toda $t$. Esta familia es creciente en el sentido de que $F_{s} \subset F_{t}$ cuando $s \leq t$. Así, una filtración puede ser vista como una estructura de información dinámica y $F_{t}$ representa toda la información disponible al tiempo $t$. El hecho de que la filtración esté aumentando significa que hay más y más información conocida conforme el tiempo transcurre y que la información pasada no se olvida. Se cumple además que $W_{t}$ tiene incrementos independientes normales con medias cero y varianzas iguales a los incrementos en el tiempo. Al tiempo $t=0$, se define $W_{0}=0$. El proceso $\mathrm{d} W_{t}$ modela las fluctuaciones propias de los rendimientos del subyacente $\mathrm{y}$, como se sabe, satisface: $\mathrm{d} W_{t}$ se distribuye $N(0, \mathrm{~d} t), \quad \mathrm{E}\left[\mathrm{d} W_{t}\right]=0$ y 
$\operatorname{Var}\left[\mathrm{d} W_{t}\right]=\mathrm{E}\left[\left(\mathrm{d} W_{t}\right)^{2}\right]=\mathrm{d} t$. Se supone que el precio del activo subyacente al tiempo $t, S_{t}$, es conducido por el movimiento geométrico Browniano

$$
\mathrm{d} S_{t}=\mu S_{t} \mathrm{~d} t+\sigma S_{t} \mathrm{~d} W_{t}
$$

En este caso, el parámetro de tendencia, $\mu \in \mathbf{R}$, representa el rendimiento medio esperado (anualizado) y $\sigma>0$ la volatilidad (anualizada). Una simple aplicación del lema de Itô (véase el Apéndice) conduce a

$$
\mathrm{d} \ln \left(S_{t}\right)=\left(\mu-\frac{1}{2} \sigma^{2}\right) \mathrm{d} t+\sigma \mathrm{d} W_{t},
$$

lo que, a su vez, implica

$$
S_{t}=S_{0} e^{\sigma W_{t}+\left(\mu-\frac{1}{2} \sigma^{2}\right) t}
$$

La expresión anterior es invertible, en el sentido que se puede despejar $W_{t}$ y esto significa que el proceso $\left(S_{t}\right)_{t \geq 0}$ es adaptado a la filtración $\left(F_{t}\right)_{t \geq 0}$.

\section{Dinámica del precio de la opción}

El valor, prima o precio de una opción europea de compra es claramente función de los distintos parámetros que intervienen en los términos o cláusulas del contrato, tales como: el precio de ejercicio $K$ y la vida del contrato $T-t$, donde $T$ es la fecha de vencimiento y $t$ es la fecha de inicio del contrato. Por supuesto, el valor de dicha opción también dependerá de las características del activo subyacente, tales como: su precio de contado (de mercado), $S_{t}$, rendimiento medio esperado, $\mu$, y volatilidad, $\sigma$, así como de la tasa de interés, $r$, que prevalece en el mercado de crédito con el fin de calcular el valor del dinero en el tiempo. Por lo anterior, se puede escribir el valor de una opción europea como

$$
c=c\left(S_{t}, t ; K, T, \sigma, \mu, r\right) .
$$


Observe que $S_{t}$ y $t$ son las variables relevantes en el contrato ( $S_{t}$ recibe, frecuentemente, el nombre de variable de estado). En lo que sigue, no se hará mención explícita de los parámetros, $K, T, \sigma, \mu \quad$ y $\quad r$, excepto cuando sea necesario. Es decir, el valor de la opción se denotará simplemente como $c=c\left(S_{t}, t\right)$. Posteriormente, se mostrará que $c$ no depende del parámetro de aversión al riesgo, $\mu$.

Durante el intervalo de tiempo $[t, t+\mathrm{d} t]$, el activo subyacente cambia marginalmente de $S_{t}$ a $S_{t}+\mathrm{d} S_{t}$, en consecuencia, el precio de la opción cambia de $c$ a $c+\mathrm{d} c$. El cambio marginal en el precio de la opción se obtiene mediante el lema de Itô, como (ver Apéndice):

$$
\mathrm{d} c=\left(\frac{\partial c}{\partial t}+\frac{\partial c}{\partial S_{t}} \mu S_{t}+\frac{1}{2} \sigma^{2} S_{t}^{2} \frac{\partial^{2} c}{\partial S_{t}^{2}}\right) \mathrm{d} t+\frac{\partial c}{\partial S_{t}} \sigma S_{t} \mathrm{~d} W_{t}
$$

Rendimiento de los activos

Se supone ahora que el individuo tiene acceso a tres activos reales: una acción de precio $S_{t}$, una opción sobre la acción de precio $c=c\left(S_{t}, t\right)$ y un bono de precio $b_{t}$ libre de riesgo de incumplimiento que paga tasa fija $r$. De acuerdo con la ecuación (1), el rendimiento que paga el activo subyacente es

$$
\mathrm{d} R_{S}=\frac{\mathrm{d} S_{t}}{S_{t}}=\mu \mathrm{d} t+\sigma \mathrm{d} W_{t}
$$

Asimismo, el rendimiento que paga el bono está dado por

$$
\mathrm{d} R_{b}=r \mathrm{~d} t
$$

En virtud de la ecuación (2), el rendimiento de la opción satisface

$$
\mathrm{d} R_{c}=\frac{\mathrm{d} c}{c}=\mu_{c} \mathrm{~d} t+\sigma_{c} \mathrm{~d} W_{t}
$$


donde

$$
\mu_{c} \equiv\left(\frac{\partial c}{\partial t}+\frac{\partial c}{\partial S_{t}} \mu S_{t}+\frac{1}{2} \frac{\partial^{2} c}{\partial S_{t}^{2}} \sigma^{2} S_{t}^{2}\right) \frac{1}{c}
$$

$\mathrm{y}$

$$
\sigma_{c}=\frac{\partial c}{\partial S_{t}} \sigma S_{t} \frac{1}{c}
$$

Se supone que el valor intrínseco (al vencimiento) de la opción está dado por $c\left(S_{t}, T\right)=\max \left(S_{t}-K, 0\right)$, donde $K$ es el precio de ejercicio de la opción.

\section{Construcción del portafolio}

Sean $\alpha_{1 t}=S_{t} / A_{t}$ la proporción de la riqueza que el individuo asigna a la tenencia de acciones, $\alpha_{2 t}=c / A_{t}$ la proporción de la riqueza que asigna a una opción europea de compra sobre la acción, y $1-\alpha_{1 t}-\alpha_{2 t}$ la fracción complementaria que se destina a un instrumento libre de riesgo que paga un rendimiento $r$ constante a cualquier plazo. En este caso, la riqueza, $A_{\mathrm{t}}$, satisface:

$$
\mathrm{d} A_{t}=A_{t}\left(1-\alpha_{1 t}-\alpha_{2 t}\right) \mathrm{d} R_{b}+A_{t} \alpha_{1 t} \mathrm{~d} R_{S}+A_{t} \alpha_{2 t} \mathrm{~d} R_{c}
$$

Después de sustituir (3), (4) y (5) en la ecuación anterior, ésta se puede reescribir como

$$
\mathrm{d} A_{t}=A_{t}\left(r+(\mu-r) \alpha_{1 t}+\left(\mu_{c}-r\right) \alpha_{2 t}\right) \mathrm{d} t+A_{t}\left(\alpha_{1 t} \sigma+\alpha_{2 t} \sigma_{c}\right) \mathrm{d} W_{t} .
$$

En este caso, se sigue que la media y la varianza del rendimiento de la riqueza satisfacen, respectivamente,

$$
\mu_{A}:=\mathrm{E}\left[\frac{\mathrm{d} A_{t}}{A_{t}}\right] \frac{1}{\mathrm{~d} t}=r+(\mu-r) \alpha_{1 t}+\left(\mu_{c}-r\right) \alpha_{2 t}
$$

$\mathrm{y}$

$$
\sigma_{A}^{2}:=\operatorname{Var}\left[\frac{\mathrm{d} A_{t}}{A_{t}}\right] \frac{1}{\mathrm{~d} t}=\left(\alpha_{1 t} \sigma+\alpha_{2 t} \sigma_{c}\right)^{2}
$$


Considere ahora el problema de decisión sobre las proporciones, $\alpha_{1 t}$ y $\alpha_{2 t}$, de la riqueza $A_{t}$, que se asignan a los diferentes activos (véase Markowitz (1952)):

$$
\begin{gathered}
\underset{\alpha_{1 t}, \alpha_{2 t}}{\operatorname{Minimizar}} \frac{1}{2} \sigma_{A}^{2}=\frac{1}{2}\left(\alpha_{1 t}, \alpha_{2 t}\right)\left(\begin{array}{cc}
\sigma^{2} & \sigma \sigma_{c} \\
\sigma \sigma_{c} & \sigma_{c}^{2}
\end{array}\right)\left(\begin{array}{l}
\alpha_{1 t} \\
\alpha_{2 t}
\end{array}\right) \\
\text { sujeto a: } \mu_{A}=r+\left(\alpha_{1 t}, \alpha_{2 t}\right)\left(\begin{array}{cc}
\mu & -r \\
\mu_{c} & -r
\end{array}\right)=\mu_{0},
\end{gathered}
$$

donde $\mu_{0}$ es un rendimiento dado. El Lagrangeano asociado al problema anterior, de programación no lineal, es

$$
\mathrm{L} \equiv \frac{1}{2}\left(\alpha_{1 t} \sigma+\alpha_{2 t} \sigma_{c}\right)^{2}+v\left[\mu_{0}-r-\alpha_{1 t}\left(\mu_{1}-r\right)-\alpha_{2 t}\left(\mu_{2}-r\right)\right]
$$

donde $v$ es el multiplicador de Lagrange asociado a la restricción. Las condiciones de primer orden $\partial \mathrm{L} / \partial \alpha_{1 t}, i=1,2$, conducen a

$$
\left\{\begin{array}{l}
\alpha_{1 t} \sigma+\alpha_{2 t} \sigma_{c}=v\left(\frac{\mu-r}{\sigma}\right), \\
\alpha_{1 t} \sigma+\alpha_{2 t} \sigma_{c}=v\left(\frac{\mu_{c}-r}{\sigma_{c}}\right)
\end{array}\right.
$$

En consecuencia,

$$
\frac{\mu-r}{\sigma}=\frac{\mu_{c}-r}{\sigma_{c}}
$$

Después de sustituir $\mu_{\mathrm{c}} \mathrm{y} \sigma_{\mathrm{c}}$, dadas en (5), en la ecuación anterior se tiene que

$$
\left(\frac{\partial c}{\partial t}+\frac{\partial c}{\partial S_{t}} \mu S_{t}+\frac{1}{2} \frac{\partial^{2} c}{\partial S_{t}^{2}} \sigma^{2} S_{t}^{2}\right)-r c=(\mu-r) \frac{\partial c}{\partial S_{t}} S_{t}
$$


lo cual conduce a la ecuación diferencial parcial de Black-Scholes-Merton (1973),

$$
\frac{\partial c}{\partial t}+\frac{\partial c}{\partial S_{t}} r S_{t}+\frac{1}{2} \frac{\partial^{2} c}{\partial S_{t}^{2}} \sigma^{2} S_{t}^{2}-r c=0
$$

junto con la condición de frontera

$$
c\left(S_{t}, T\right)=\max \left(S_{t}-K, 0\right) .
$$

Dado que la ecuación diferencial parcial anterior es de segundo grado, se requiere otra condición dada por $c(0, t)=0$, la cual dice que si el activo subyacente es libre (gratis) la opción carece de valor.

Observe también que la ecuación (8) es una ecuación diferencial parcial lineal parabólica. De hecho, casi todas las ecuaciones diferenciales parciales en matemáticas financieras tienen una forma similar. La linealidad significa que si se tienen dos soluciones, entonces la suma de ellas también es una solución. En otras palabras, si todos los activos de un portafolio satisfacen la ecuación (8), entonces el portafolio también la satisface. Por último, el hecho de que la ecuación diferencial parcial sea parabólica significa que está relacionada con la ecuación de difusión de calor. Esta ecuación describe cómo se difunde el calor en una viga (hecha de algún material conductor) de dimensión infinita. En este caso, la temperatura en un punto de la viga está asociada al rendimiento del activo.

La ecuación de Black-Scholes-Merton contiene todas las variables que determinan el valor del contrato y los parámetros tales como el precio de contado del activo subyacente, el tiempo y la volatilidad, pero no se hace mención al rendimiento medio esperado $\mu$. Cualquier dependencia sobre $\mu$ se ha eliminado al anular el coeficiente de $\mathrm{d} W_{t}$ en el cambio de valor del portafolio. Observe que en su lugar aparece, en la ecuación (8), la tasa de interés libre de riesgo, $r$. Esto significa que si todos los participantes en el mercado de opciones están de acuerdo con el nivel de volatilidad de los rendimientos del activo, entonces están igualmente de acuerdo con el valor de la opción aunque tengan diferentes preferencias al riesgo expresadas a través de $\mu$. En otras palabras, todos los agentes están dispuestos a omitir sus preferencias al riesgo, $\mu$, y aceptar un rendimiento libre de riesgo, $r$, después de ponerse de acuerdo con el nivel de volatilidad del activo subyacente. 
Obtención de la ecuación BSM mediante argumentos del modelo CAPM

A continuación se obtiene la ecuación Black-Scholes-Merton utilizando el modelo CAPM, el cual se obtiene de la ecuación (7). El modelo CAPM se utiliza con mucha frecuencia para valuar acciones bajo condiciones de equilibrio de mercado. El modelo CAPM describe la relación entre el riesgo y el rendimiento esperado de un activo y es muy útil para valuar los títulos de capital que emiten las empresas para financiarse. Específicamente, el modelo CAPM dice que la diferencia entre el rendimiento de una acción y una tasa libre de riesgo de incumplimiento (por ejemplo, la tasa de CETES, Certificados de la Tesorería) es proporcional a la diferencia entre el rendimiento del mercado (por ejemplo, el Índice de Precios y Cotizaciones de la Bolsa Mexicana de Valores) y la tasa libre de riesgo (de CETES).

Suponga, como antes, que la dinámica del precio del activo subyacente es conducida por el movimiento geométrico Browniano. De esta manera, el rendimiento del activo es

$$
\mathrm{d} R_{S}=\frac{\mathrm{d} S_{t}}{S_{t}}=\mu \mathrm{d} t+\sigma \mathrm{d} W_{t}
$$

Para calcular el cambio en el precio de la opción por cambios en $S_{t}$ se utiliza la expansión en serie de Taylor hasta términos de segundo orden:

$$
\begin{aligned}
\mathrm{d} c & =\frac{\partial c}{\partial S_{t}} \mathrm{~d} S_{t}+\frac{\partial c}{\partial t} \mathrm{~d} t+\frac{1}{2} \frac{\partial^{2} c}{\partial S_{t}^{2}} \sigma^{2} S_{t}^{2} \mathrm{~d} t \\
& =\left(\frac{\partial c}{\partial S_{t}} \mu S_{t}+\frac{\partial c}{\partial t}+\frac{1}{2} \frac{\partial^{2} c}{\partial S_{t}^{2}} \sigma^{2} S_{t}^{2}\right) \mathrm{d} t+\frac{\partial c}{\partial S_{t}} \sigma S_{t} \mathrm{~d} W .
\end{aligned}
$$

En este caso, el rendimiento de la opción está dado por

$$
\mathrm{d} R_{c}=\frac{\mathrm{d} c}{c}=\frac{\partial c}{\partial S_{t}} \mathrm{~d} R_{S} \frac{S_{t}}{c}+\frac{1}{c}\left(\frac{\partial c}{\partial t}+\frac{1}{2} \sigma^{2} S_{t}^{2} \frac{\partial^{2} c}{\partial S_{t}^{2}}\right) \mathrm{d} t
$$

De acuerdo con el modelo CAPM, los rendimientos de la opción y del activo subyacente satisfacen, respectivamente, las siguientes relaciones lineales con respecto del rendimiento del mercado, $\mathrm{d} R_{M}$, 


$$
\mathrm{E}\left[\mathrm{d} R_{c}\right]-r \mathrm{~d} t=\beta_{c}\left[\mathrm{E}\left[\mathrm{d} R_{M}\right]-r \mathrm{~d} t\right]
$$

y

$$
\mathrm{E}\left[\mathrm{d} R_{S}\right]-r \mathrm{~d} t=\beta_{S}\left[\mathrm{E}\left[\mathrm{d} R_{M}\right]-r \mathrm{~d} t\right]
$$

donde

$$
\beta_{S}=\frac{\operatorname{Cov}\left(\mathrm{d} R_{S}, \mathrm{~d} R_{M}\right)}{\operatorname{Var}\left(\mathrm{d} R_{M}\right)}
$$

$\mathrm{y}$

$$
\begin{aligned}
\beta_{c} & =\frac{\operatorname{Cov}\left(\mathrm{d} R_{c}, \mathrm{~d} R_{M}\right)}{\operatorname{Var}\left(\mathrm{d} R_{M}\right)} \\
& =\frac{\operatorname{Cov}\left(\frac{\partial c}{\partial S_{t}} \mathrm{~d} R_{S} \frac{S_{t}}{c}, \mathrm{~d} R_{M}\right)}{\operatorname{Var}\left(\mathrm{d} R_{M}\right)} \\
& =\frac{\partial c}{\partial S_{t}} \frac{S_{t}}{c} \frac{\operatorname{Cov}\left(\mathrm{d} R_{S}, \mathrm{~d} R_{M}\right)}{\operatorname{Var}\left(R_{M}\right)} \\
& =\frac{\partial c}{\partial S_{t}} \frac{S_{t}}{c} \beta_{S},
\end{aligned}
$$

porque $\operatorname{Cov}\left(\mathrm{d} t, \mathrm{~d} R_{M}\right)=0$. Si se sustituyen las ecuaciones (11), (13), (14) y (15) en (12), se tiene

$$
\begin{array}{rl}
\mathrm{E}\left[\frac{\mathrm{d} c}{c}\right]-r \mathrm{~d} & t=\beta_{c}\left(\mathrm{E}\left[\mathrm{d} R_{M}\right]-r \mathrm{~d} t\right) \\
& =\frac{\partial c}{\partial S_{t}} \frac{S_{t}}{c} \beta_{c}\left(\mathrm{E}\left[\mathrm{d} R_{M}\right]-r \mathrm{~d} t\right) \\
& =\frac{\partial c}{\partial S_{t}} \frac{S_{t}}{c} \beta_{S}\left(\frac{1}{\beta_{S}}\left(\mathrm{E}\left[\mathrm{d} R_{S}\right]-r \mathrm{~d} t\right)\right) \\
& =\frac{\partial c}{\partial S_{t}} \frac{S_{t}}{c}\left(\mathrm{E}\left[\mathrm{d} R_{S}\right]-r \mathrm{~d} t\right)
\end{array}
$$


lo cual implica que

$$
\mathrm{E}[\mathrm{d} c]-r c \mathrm{~d} t=\frac{\partial c}{\partial S_{t}} S_{t}\left(\mathrm{E}\left[\mathrm{d} R_{S}\right]-r \mathrm{~d} t\right)
$$

Si se sustituye la ecuación (9) en (17), se sigue que

$$
\begin{aligned}
\mathrm{E}[\mathrm{d} c]-r c \mathrm{~d} t & =\frac{\partial c}{\partial S_{t}} S_{t}\left(\mathrm{E}\left[\mathrm{d} R_{S}\right]-r \mathrm{~d} t\right) \\
= & \frac{\partial c}{\partial S_{t}} S_{t}\left(\mathrm{E}\left[\frac{\mathrm{d} S_{t}}{S_{t}}\right]-r \mathrm{~d} t\right) \\
& =\frac{\partial c}{\partial S_{t}} S_{t}(\mathrm{E}[\mu \mathrm{d} t+\sigma \mathrm{d} W]-r \mathrm{~d} t) \\
& =\frac{\partial c}{\partial S_{t}} S_{t}(\mu-r) \mathrm{d} t,
\end{aligned}
$$

donde se ha considerado que $\mathrm{E}\left[\mathrm{d} W_{t}\right]=0$. Por último, si se sustituye (10) en la ecuación (18), se tiene

$$
\mathrm{E}\left[\left(\frac{\partial c}{\partial S_{t}} \mu S_{t}+\frac{\partial c}{\partial t}+\frac{1}{2} \frac{\partial^{2} c}{\partial S_{t}^{2}} \sigma^{2} S_{t}^{2}\right) \mathrm{d} t+\frac{\partial c}{\partial S_{t}} \sigma S_{t} \mathrm{~d} W\right]-r c \mathrm{~d} t=\frac{\partial c}{\partial S_{t}} S_{t}(\mu-r) \mathrm{d} t
$$

equivalentemente

$$
\frac{\partial c}{\partial t}+r S_{t} \frac{\partial c}{\partial S_{t}}+\frac{1}{2} \sigma^{2} S_{t}^{2} \frac{\partial^{2} c}{\partial S_{t}^{2}}-r c=0
$$

Este resultado coincide con (8). 


\section{Obtención de la ecuación de BSM mediante el postulado de racionalización económica}

Esta sección muestra la consistencia entre la existencia de consumidores racionales con los resultados derivados de los modelos de Markowitz y CAPM. Se supone como antes que el individuo tiene acceso a tres activos reales: una acción de precio $S_{t}$, una opción sobre la acción de precio $c=c\left(S_{t}, t\right)$ y un bono de precio $b_{t}$ libre de riesgo de incumplimiento que paga tasa fija $r$. Suponga que el rendimiento que paga el activo subyacente es

$$
\mathrm{d} R_{S}=\mu \mathrm{d} t+\sigma \mathrm{d} W_{t}
$$

Asimismo, suponga que el rendimiento que paga el bono está dado por

$$
\mathrm{d} R_{b}=r \mathrm{~d} t \text {. }
$$

En vista de (20), la aplicación del lema de Itô a $c=c\left(S_{t}, t\right)$ conduce a que el rendimiento de la opción satisface

$$
\mathrm{d} R_{c}=\frac{\mathrm{d} c}{c}=\mu_{c} \mathrm{~d} t+\sigma_{c} \mathrm{~d} W_{t}
$$

donde

$$
\mu_{c} \equiv\left(\frac{\partial c}{\partial t}+\frac{\partial c}{\partial S_{t}} \mu S_{t}+\frac{1}{2} \frac{\partial^{2} c}{\partial S_{t}^{2}} \sigma^{2} S_{t}^{2}\right) \frac{1}{c}
$$

$\mathrm{y}$

$$
\sigma_{c} \equiv \frac{\partial c}{\partial S_{t}} \sigma S_{t} \frac{1}{c}
$$

Se supone que $c\left(S_{t}, T\right)=\max \left(S_{t}-K, 0\right)$, donde $K$ es el precio de ejercicio de la opción. Sean $\omega_{1 t}=S_{t} / A_{t}$ la proporción de la riqueza que el individuo asigna a una opción europea de compra sobre la acción, $\omega_{2 t}=c / a_{t}$ la proporción de la riqueza que asigna a una opción europea de compra sobre la acción, y $1-\omega_{1 t}-\omega_{2 t}$ la fracción complementaria que se asigna a un instrumento libre de riesgo que paga 
un rendimiento $r$ constante a cualquier plazo. La variable $\omega_{i t}$ es diferente de $\alpha_{i t}$, la cual fue introducida en la sección anterior en (6) porque $\omega_{i t}$ incorpora la decisión de consumo. Por la misma razón, la $A_{t}$ de (6) y la $a_{t}$ de (24) son diferentes. En este caso, el agente desea resolver el siguiente problema:

$$
\underset{C_{t}, \omega_{1 t}, \omega_{2 t}}{\operatorname{Maximizar}} \mathrm{E}\left[\int_{0}^{T} \frac{C_{S}^{\gamma}}{\gamma} e^{-\delta s} \mathrm{~d} s+b\left(a_{T}, T\right) \mid F_{t}\right]
$$

sujeto a:

$$
\mathrm{d} a_{t}=a_{t} \omega_{1 t} \mathrm{~d} R_{S}+a_{t} \omega_{2 t} \mathrm{~d} R_{c}+a_{t}\left(1-\omega_{1 t}-\omega_{2 t}\right) \mathrm{d} R_{b}-C_{t} \mathrm{~d} t,
$$

donde $C_{t}$ es consumo, $\gamma$ es un parámetro de preferencias (grado de aversión al riesgo), $\delta$ es la tasa subjetiva de descuento (entre mayor es $\delta$, más ansioso está el consumidor por el consumo presente), $F_{t}$ es la información relevante al tiempo $t, \mathrm{y}$

$$
b\left(a_{T}, T\right)=\frac{a T^{\gamma}}{\gamma} e^{-\delta T}
$$

representa una herencia. Después de sustituir (20), (21) y (22) en la restricción presupuestal, ésta se puede reescribir como

$$
\mathrm{d} a_{t}=a_{t}\left(r+(\mu-r) \omega_{1 t}+\left(\mu_{c}-r\right) \omega_{2 t}-\frac{C_{t}}{a_{t}}\right) \mathrm{d} t+a_{t}\left(\omega_{1 t} \sigma+\omega_{2 t} \sigma_{c}\right) \mathrm{d} W_{t}
$$

Si se define (la función de utilidad indirecta)

$$
J\left(a_{t}, t\right)=\max _{C_{t}, \omega_{1 t}, \omega_{2 t}} \mathrm{E}\left[\int_{t}^{T} \frac{C_{s}^{\gamma}}{\gamma} e^{-\delta s} \mathrm{~d} s+b\left(a_{T}, T\right) \mid F_{t}\right]
$$


y se aplica la recursividad de $J$, el teorema del valor medio del cálculo integral y una expansión en serie de Taylor hasta términos de primer orden se sigue que

$$
\begin{aligned}
J\left(a_{t}, t\right) & =\max _{C_{t}, \omega_{1 t}, \omega_{2 t}} \mathrm{E}\left[\int_{t}^{t+\mathrm{d} t} \frac{C_{s}^{\gamma}}{\gamma} e^{-\delta s} \mathrm{~d} s+\int_{t+\mathrm{d} t}^{T} \frac{C_{s}^{\gamma}}{\gamma} e^{-\delta s} \mathrm{~d} s+b\left(a_{T}, T\right) \mid F_{t}\right] \\
& =\max _{C_{t}, \omega_{1 t}, \omega_{2} t[[t, t+d t]} \mathrm{E}\left[\int_{t}^{t+\mathrm{d} t} \frac{C_{s}^{\gamma}}{\gamma} e^{-\delta s} \mathrm{~d} s+J\left(a_{t}+\mathrm{d} a_{t}, t+\mathrm{d} t\right) \mid F_{t}\right] \\
& =\max _{C_{t}, \omega_{1 t}, \omega_{2 t}[[t, t+d t]} \mathrm{E}\left[\frac{C_{s}^{\gamma}}{\gamma} e^{-\delta t} \mathrm{~d} t+o(\mathrm{~d} t)+J\left(a_{t}, t\right)+\mathrm{d} J\left(a_{t}, t\right) \mid F_{t}\right] .
\end{aligned}
$$

Observe también que en la frontera, i.e, en $T$, se sigue que $J\left(a_{T}, T\right)=b\left(a_{T}, T\right)$. En virtud del lema de Itô, aplicado a $J=J\left(a_{t}, t\right)$, se cumple que

$$
\begin{aligned}
0 & =\max _{C_{t}, \omega_{t, t}, \omega_{2 t}} \mathrm{E}\left[\frac{C_{t}^{\gamma}}{\gamma} e^{-\delta t} \mathrm{~d} t+o(\mathrm{~d} t)+\left[J_{t}+J_{a} a_{t}\left(r+(\mu-r) \omega_{1 t}+\left(\mu_{c}-r\right) \omega_{2 t}-\frac{C_{t}}{a_{t}}\right)\right.\right. \\
& \left.\left.+\frac{1}{2} J_{a a} a_{t}^{2}\left(\omega_{1 t} \sigma+\omega_{2 t} \sigma_{c}\right)^{2}\right] \mathrm{~d} t+J_{a} a_{t}\left(\omega_{1 t} \sigma+\omega_{2 t} \sigma_{c}\right) \mathrm{d} W_{t} \mid F_{t}\right]
\end{aligned}
$$

donde $o(\mathrm{~d} t) / \mathrm{d} t \rightarrow 0$ si $\mathrm{d} t \rightarrow 0$. Si se toman esperanzas de los términos dentro del paréntesis $\mathrm{y}$, posteriormente, se divide entre $\mathrm{d} t$ y se toma el límite cuando $\mathrm{d} t \rightarrow 0$, se sigue que

$$
\begin{aligned}
0 & =\max _{C_{t}, \omega_{1 t}, \omega_{2 t}}\left\{\frac{C_{t}^{\gamma}}{\gamma} e^{-\delta t}+J_{t}+J_{a} a_{t}\left(r+(\mu-r) \omega_{1 t}+\left(\mu_{c}-r\right) \omega_{2 t}-\frac{C_{t}}{a_{t}}\right)\right. \\
& \left.+\frac{1}{2} J_{a a} a_{t}^{2}\left(\omega_{1 t} \sigma+\omega_{2 t} \sigma_{c}\right)^{2}\right\} .
\end{aligned}
$$


Considere un candidato de solución de la ecuación anterior de la forma (con variables separables, es decir, con dos factores uno que depende de $a_{t} \mathrm{y}$ otro de $t$ )

$$
J\left(a_{t}, t\right)=V\left(a_{t}\right) e^{-\delta t},
$$

Entonces

$$
J_{a}=V^{\prime}\left(a_{t}\right) e^{-\delta t}, \quad J_{a a}=V^{\prime \prime}\left(a_{t}\right) e^{-\delta t} \text { y } J_{t}=-\delta V\left(a_{t}\right) e^{-\delta t} .
$$

Ahora bien, si $C_{t}, \omega_{1 t}$ y $\omega_{2 t}$ son óptimos, se obtiene que

$$
\begin{aligned}
0= & \frac{C_{t}^{\gamma}}{\gamma}-\delta V\left(a_{t}\right)+V^{\prime}\left(a_{t}\right) a_{t}\left(r+(\mu-r) \omega_{1 t}+\left(\mu_{c}-r\right) \omega_{2 t}-\frac{C_{t}}{a_{t}}\right) \\
& +\frac{1}{2} V^{\prime \prime}\left(a_{t}\right) a_{t}^{2}\left(\omega_{1 t} \sigma+\omega_{2 t} \sigma_{c}\right)^{2} .
\end{aligned}
$$

Suponga que la función $V\left(a_{t}\right)$ es de la forma

$$
V\left(a_{t}\right)=\beta \frac{a_{t}^{\gamma}}{\gamma},
$$

donde $\beta$ tiene que ser determinada a partir de (27), entonces

$$
V^{\prime}\left(a_{t}\right)=\beta a_{t}^{\gamma-1} \text { y } V^{\prime \prime}\left(a_{t}\right)=\beta(\gamma-1) a_{t}^{\gamma-2} \text {. }
$$

De esta manera, la ecuación (27) se transforma en

$$
\begin{aligned}
0= & \frac{C_{t}^{\gamma}}{\gamma}-\delta \beta \frac{a_{t}^{\gamma}}{\gamma}+\beta a_{t}^{\gamma}\left(r+(\mu-r) \omega_{1 t}+\left(\mu_{c}-r\right) \omega_{2 t}-\frac{C_{t}}{a_{t}}\right) \\
& +\frac{1}{2} \beta(\gamma-1) a_{t}^{\gamma}\left(\omega_{1 t} \sigma+\omega_{2 t} \sigma_{c}\right)^{2} .
\end{aligned}
$$


Al derivar la expresión (28) con respecto de $C_{t}, \omega_{1 t}$ y $\omega_{2 t}$, se obtienen, respectivamente:

$$
\begin{gathered}
C_{t}^{\gamma-1}-\beta a_{t}^{\gamma-1}=0, \\
\beta a_{t}^{\gamma}(\mu-r)+\beta(\gamma-1) a_{t}^{\gamma}\left(\omega_{1 t} \sigma+\omega_{2 t} \sigma_{c}\right) \sigma=0
\end{gathered}
$$

$\mathrm{y}$

$$
\beta a_{t}^{\gamma}\left(\mu_{c}-r\right)+\beta(\gamma-1) a_{t}^{\gamma}\left(\omega_{1 t} \sigma+\omega_{2 t} \sigma_{c}\right) \sigma_{c}=0 .
$$

Estas tres ecuaciones se pueden reescribir como:

$$
\begin{gathered}
C_{t}=\beta^{1 /(\gamma-1)} a_{t}, \\
\mu-r=(1-\gamma)\left(\omega_{1 t} \sigma+\omega_{2 t} \sigma_{c}\right) \sigma
\end{gathered}
$$

y

$$
\mu_{c}-r=(1-\gamma)\left(\omega_{1 t} \sigma+\omega_{2 t} \sigma_{c}\right) \sigma_{c}
$$

La primera ecuación indica que el consumo es proporcional al nivel de la riqueza en todo momento. Las dos últimas ecuaciones implican que los premios al riesgo de $S_{t}$ y $c\left(S_{t}, t\right)$ son iguales, es decir,

$$
\frac{\mu_{c}-r}{\sigma_{c}}=\frac{\mu-r}{\sigma} .
$$

Después de sustituir $\mu_{c}$ y $\sigma_{c}$ en la ecuación (32), se tiene que

$$
\left(\frac{\partial c}{\partial t}+\frac{\partial c}{\partial S_{t}} \mu S_{t}+\frac{1}{2} \frac{\partial^{2} c}{\partial S_{t}^{2}} \sigma^{2} S_{t}^{2}\right)-r c=(\mu-r) \frac{\partial c}{\partial S_{t}} S_{t}
$$

lo cual conduce a la ecuación diferencial parcial de Black-Scholes:

$$
\frac{\partial c}{\partial t}+\frac{\partial c}{\partial S_{t}} r S_{t}+\frac{1}{2} \frac{\partial^{2} c}{\partial S_{t}^{2}} \sigma^{2} S_{t}^{2}-r c=0
$$


junto con la condición de frontera $c\left(S_{t}, t\right)=\max \left(S_{t}-K, 0\right)$. Este resultado coincide plenamente con el obtenido en la sección anterior.

\section{Conclusiones}

Este trabajo ha mostrado que la existencia de consumidores racionales es consistente con la existencia de inversionistas minimizadores de varianza. Esto se podría traducir a que el postulado de Markowitz conlleva implícitamente la noción de racionalidad económica. Esto trae de nuevo al centro de la discusión el viejo debate sobre el comportamiento de los agentes en el proceso de toma de decisiones ¿Realmente los agentes actúan de acuerdo con estos principios o al menos en alguna forma relajada de dichos principios?

El postulado de racionalidad económica ha sido, desde hace varias décadas, un asunto de continuo debate sobre el comportamiento de los agentes económicos cuando éstos toman decisiones de consumo y portafolio. Se dice que un consumidor es racional si resuelve problemas de optimización a través de un proceso instantáneo que se lleva a cabo desde su cerebro. Evidentemente, no ha sido fácil aceptar que los consumidores cuando toman decisiones de consumo y portafolio, lo hacen de tal manera que maximizan una función de utilidad sujetos a su restricción presupuestal porque esto involucra un proceso complejo de abstracción para plantear problemas de optimización y otro proceso todavía más elaborado para resolverlos de manera instantánea. Peor aún, cuando la toma de decisiones se realiza en un ambiente de riesgo e incertidumbre, entonces el planteamiento y la resolución del problema de optimización se convierte en un asunto muy sofisticado.

Desde hace muchas décadasse han hecho varios intentos para relajar el concepto de racionalidad económica perfecta (o hiper-racionalidad). Uno de los intentos más importantes se refiere al trabajo pionero de Herbert Simon (1957) quien ya planteaba la noción de racionalidad limitada (o acotada) en la que, primero, el individuo elige una función de utilidad entre un conjunto de funciones disponibles en su cerebro y, posteriormente, ordena en términos de sus preferencias las posibles alternativas siguiendo un proceso que no es tan "fino" como el que exigirían las condiciones de primer orden de un problema de optimización, siendo el ordenamiento de posibilidades más bien un proceso "burdo" en el sentido que revisa grupos de alternativas y elige, casi instantáneamente, aquellas que pudieran estar cerca del óptimo. Por otro lado, la "economía experimental" ha logrado un avance 
impresionante, desde el trabajo seminal de Vernon Smith (1962), mostrando con experimentos conducidos la ausencia del comportamiento racional "perfecto" en los agentes. De aquí se puede concluir que el postulado de Markowitz conlleva, implícitamente, la racionalidad económica en alguna de sus formas relajadas.

Por último, es importante destacar que aunque el problema del consumidor-inversionista racional se resolvió utilizando una forma funcional específica del índice de satisfacción, se puede demostrar que la obtención de la ecuación diferencial parcial de BSM es independiente de la función de utilidad.

\section{Apéndice. El lema de Itô}

Considere una función arbitraria $f\left(S_{t}, t\right)$ donde $S_{t}$ sigue una ecuación diferencial estocástica de la forma $\mathrm{d} S_{t}=\mu S_{t} \mathrm{~d} t+\sigma S_{t} \mathrm{~d} W_{t}$, entonces la diferencial estocástica de $y, \mathrm{~d} y$, se obtiene mediante la expresión:

$$
\mathrm{d} y=\left(\frac{\partial c}{\partial t}+\frac{\partial c}{\partial S_{t}} \mu S_{t}+\frac{1}{2} \sigma{ }^{2} S_{t}^{2} \frac{\partial^{2} y}{\partial S_{t}^{2}}\right) \mathrm{d} t+\frac{\partial y}{\partial S_{t}} \sigma S_{t} \mathrm{~d} W_{t}
$$

\section{Referencias}

Black, F. y M. Scholes (1973). "The Pricing of Options and Corporate Liabilities". Journal of Political Economy, Vol.81, No.3, pp. 637-654.

Markowitz, H. M. (1952). "Portfolio selection", Journal of Finance, Vol. 7, No. 1, pp. 77-91.

Marshall, A. (1916). Principles of Economics, An Introductory Volume, Seventh edition, London: Mac Millan.

Merton, R., C. (1973). "Theory of Rational Option Pricing”. Bell Journal of Economics, Vo. 4, No. 1, pp. 141-183.

Pareto, W. (1897). "Cours d' Economie Politique”, Oeuvres Complétes. Vol 1, Genéve: Droz. 
Sharpe, W. F. (1964). "Capital Asset Prices: A Theory of Market Equilibrium Ander Conditions of Risk". Journal of Finance, Vol. 19, No. 3, pp. 425-442.

Simon, H. (1957). "A Behavioral Model of Racional Choice”. Models of Man, Social and Rational: Mathematical Essays on Rational Human Behavior in a Social Setting. New York: Wiley.

Smith, V. L. (1962). "An Experimentas Study of Competitive Market Behavior". Journal of Economy, Vol. 70, No. 2, pp. 111-137.

Venegas-Martínez, F. (2001). "Temporary Stabilization: A Stochastic Analysis". Journal of Economic Dynamics and Control, Vol. 25, No. 9, pp. 14291449.

(2005). "Bayesian Inference, Prior Information on Volatility, and Option Pricing: A Maximum Entropy Approach". International Journal of Theoretical and Applied Finance, Vol. 8, No. 1, pp.1-12.

(2006). "Stochastic Temporary Stabilization: Undiversifiable Devaluation and Income Risks". Economic Modelling, Vol.23, No.1, pp. 157173.

(2008). "Temporary Stabilization in Developing Counties and the Real Option of Waiting when Consumption Can Be Delayed". International Journal of Economic Research, Vol. 5, No.1. forthcoming.

Walras, L. (1952). Éléments d'économie Politique Pure. Paris: Librairie de Droit et de Jurisprudente. 